\title{
Unilateral intact twin tubal pregnancy in an intrauterine contraceptive device user: a case report
}

\begin{abstract}
There is a trend of rising incidence of ectopic pregnancy in recent years reaching about one in 100 pregnancies. However, the incidence of unilateral twin ectopic pregnancy is a rare occurrence. We report a case of twin ectopic pregnancy discovered early at two months of amenorrhea in 32 years old women gravida 4 presented to the emergency unit with lower abdominal pain, positive urinary BHCG and large right adnexal mass on transvaginal ultrasound. The patient was current intrauterine contraceptive device user. This case was managed surgically by salpingectomy for fear of tubal rupture. The tube contained twin gestational sacs. The crown-rump length of the embryos was 3.4 $\mathrm{cm}$. The patient's postoperative period was uneventful, and she was discharged three days after her surgery had occurred.
\end{abstract}

Keywords: ectopic pregnancy, twins, maternal morbidity
Volume 10 Issue 2 - 2019

\author{
Ahmed Samy,' Ahmed M Abbas, ${ }^{2}$ Shymaa S \\ Ali, ${ }^{3}$ Marwa Abdalla' \\ 'Department of Obstetrics and Gynaecology, Faculty of \\ Medicine, Cairo University, Egypt \\ ${ }^{2}$ Department of Obstetrics and Gynaecology, Faculty of \\ Medicine, Assiut University, Egypt \\ ${ }^{3}$ Department of Obstetrics and Gynaecology, Faculty of \\ Medicine, Suez University, Egypt
}

Correspondence: Ahmed M Abbas. MD Department of Obstetrics and Gynaecology, Assiut University, Women Health Hospital,Assiut Egypt, 7| I I I,Tel +208824| 46 I6, 20 10033851833, Fax+20889202503, Email bmr90@hotmail.com

\section{Introduction}

Ectopic pregnancy is considered the leading cause of maternal mortality in the first trimester of pregnancy in the United States, occurring in about $2 \%$ of pregnancies. ${ }^{1}$ An ectopic pregnancy occurs when a blastocyst implanted in another site away from the endometrial lining of the uterus. ${ }^{2}$ The marked rise in the incidence of ectopic pregnancy in recent years attributed to the assisted reproductive techniques and tuboplasty. ${ }^{3}$

Fallopian tubes are considered the commonest site for ectopic pregnancy is approximately $95 \%$ of cases followed by the ovaries in $3 \%$ and $(<1 \%)$ is abdominal or cervical or corneal pregnancy. ${ }^{4}$

Twin ectopic pregnancy is a rare event. Its incidence is about 1 in 20,000 spontaneous pregnancies. ${ }^{3}$ Missed or late diagnosis can end with tubal rupture, hemoperitoneum and subsequent hypovolemic shock. ${ }^{5}$

This report presents a case of unilateral intact twin ectopic pregnancy diagnosed at ten weeks in an intrauterine contraceptive device (IUCD) user.

\section{Case presentation}

A 32-year-old woman gravida $4 \mathrm{P} 3+0$ presented to the emergency unit with lower abdominal pain after two months of amenorrhea. She was using IUCD, and her previous menstrual cycles were regular, but she is not sure from her $1^{\text {st }}$ day of last menstrual period. Urinary BHCG is positive. Abdominal examination revealed mild right abdominal tenderness. Bimanual examination showed bulky uterus and a tender adnexal mass was tipped in the right fornix

Transvaginal ultrasound (TVUS) was obtained revealing an IUCD without evidence of an intrauterine gestational sac. However, there was large right adnexal mass measuring $7 \mathrm{~cm}$ with mild intrapelvic fluid collection.

These findings were interpreted to represent a case of right ectopic pregnancy. The patient was prepared for the termination of pregnancy by laparotomy. Her hemoglobin $(\mathrm{Hg})$ level was $9 \mathrm{~g} / \mathrm{dL}$.
Laparotomy through a Pfannenstiel incision was performed under spinal anesthesia after counseling the patient about her current condition and obtaining an informed consent from her to carry out the procedure. There was mild hemoperitoneum with normal uterus and left adnexa. Right fallopian tube was markedly distended through its whole length, but interestingly it was intact with no evidence of any rupture in the tubal wall. Right salpingectomy was performed. The tube contained two embryos in two separate gestational sacs. No intra-operative blood transfusion. The patient had an excellent postoperative course and was discharged on day 3 post-operation (Figure $1 \& 2$ ).

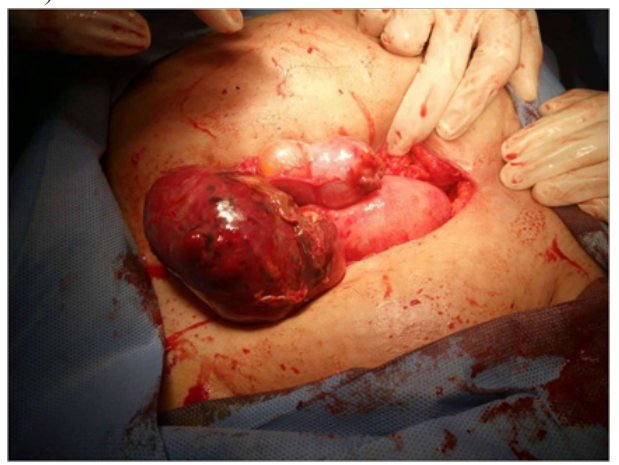

Figure I Markedly distended intact right fallopian tube intra-operatively.

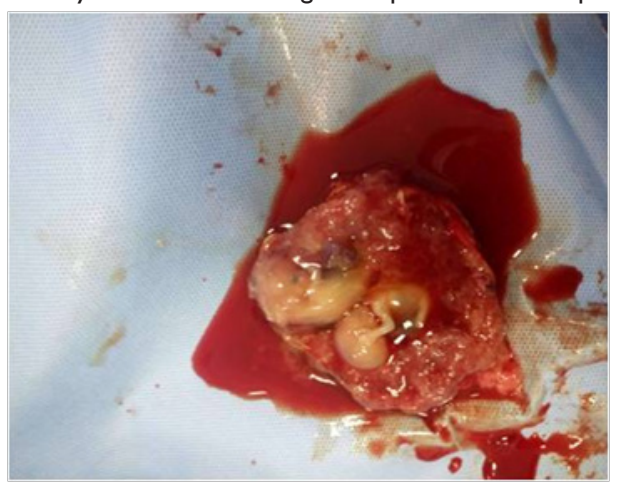

Figure 2 Twin gestational sacs after salpingectomy. 


\section{Discussion}

The frequency of ectopic pregnancies has been increasing since the 1970s. The most common risk factors related to the rising incidence of ectopic pregnancy are: a history of tubal surgery, pelvic inflammatory disease, previous ectopic pregnancy, and use of ovulation induction drugs or assisted reproductive technology. Additionally, the use of an IUCD, advanced age, smoking, and congenital uterine anomalies are minor risk factors. ${ }^{6}$

Classically, ectopic pregnancy is presented by a clinical triad consists of pain; amenorrhea and vaginal bleeding. ${ }^{7}$ Live twin ectopics pregnancies are very rare. The first case of live twin ectopics pregnancy was described in $1994 .^{2}$ However, De Ott was firstly described the occurrence of unilateral twin ectopic pregnancy in 1891. ${ }^{8}$ Monochorionic, monoamniotic twin pregnancies will be unilateral. On the other hand, dichorionic diamniotic twin pregnancies are possibly unilateral but may rarely present as a bilateral ectopic. ${ }^{9}$

TVUS has become the method of choice for evaluating early pregnancy complications due to its high accuracy related to its high resolution. TVUS permits visualization of an ectopic mass with or without an embryonic pole within it on a meticulous evaluation of the adnexa of patients suspected of having ectopic pregnancies. ${ }^{3}$

The key to diagnosing an ectopic pregnancy is the presence or absence of an intrauterine gestational sac with a correlation of serum BHCG levels. ${ }^{10}$ Indirect signs such as fluid in the Douglas Pouch, free fluid in the pelvis or a pseudo sac in the endometrium could help establish the diagnosis. ${ }^{9}$

Factors that affect the line of treatment of an ectopic pregnancy are the clinical presentation, size, and BHCG levels. It may require conservative, medical or surgical intervention. Surgical treatment is indicated in those who have contraindications to medical treatment or refuse its use and those in whom medical treatment has failed or patients who are hemodynamically unstable at presentation. ${ }^{9}$

Medical treatment with methotrexate given intramuscular or locally under ultrasound guidance has been reported in a few cases. Treatment algorithm for medical management of ectopic pregnancy is based on the size measured by ultrasound. ${ }^{10}$

In most of the reported cases of twin ectopic pregnancy, surgical management was mandatory due to the large size of the ectopic pregnancy. ${ }^{11}$ The large size of the ectopic pregnancy in the present case warranted surgical management. Surgical intervention in our case revealed distended right fallopian tube through its entire length without evidence of tubal rupture. A similar finding has been reported in other cases of twin ectopic pregnancy previously published in the literature. ${ }^{11}$

From our surgical findings, tubal rupture may be attributed to the trophoblastic invasion which increases with increasing gestational age, not due to tubal distension. Although a twin pregnancy may be larger, trophoblastic invasion may be less due to an earlier gestational age, compared with an equivalently sized singleton pregnancy.
Therefore it is less liable to rupture. This explains why tubal ruptured is less liable to occur on twin ectopic pregnancy despite more tubal distension.

Salpingectomy is the recommended surgical line of treatment; however, salpingostomy can be considered for patients with one tube who are wishing to preserve their fertility. ${ }^{12}$ In our case salpingectomy was done as the woman's fertility will not be affected markedly.

\section{Acknowledgments}

None.

\section{Conflicts of interest}

The authors have no conflicts of interest to declare.

\section{Ethical approval}

Written informed consent was obtained from the patient for publication of the details and images of her case.

\section{References}

1. George M, Nadarajah S, Ong CL. Unilateral twin ectopic pregnancy. JHK Coll Radiol. 2010;12:186-189.

2. Ghike S, Somalwar S, Mitra K, et al. A unilateral twin ectopic pregnancy (Diamniotic-Dichorionic): a rare case. $J$ South Asian Federation Obstet Gynaecol. 2011;3(2):103-105.

3. Rolle CJ, Wai CY, Bawdon R, et al. Unilateral twin ectopic pregnancy in a patient with a history of multiple sexually transmitted infections. Infect Dis Obstet Gynecol. 2006;2006:10306.

4. Goswami D, Rathore AM, Batra S, et al. Facility-based review of 296 maternal deaths at a tertiary centre in India: Could they be prevented? $J$ Obstet Gynaecol Res. 2013;39(12):1569-1579.

5. Chang J, Elam-Evans LD, Berg CJ, et al. Pregnancy related mortality surveillance-United States, 1991-1999. MMWR Surveill Summ. 2003;52(2):1-9.

6. Teresa T, Ali MD, Khazaei MD. Spontaneous unilateral dizygotic twin tubal pregnancy. J Clin Ultrasound. 2009;37(2):104-106.

7. Ectopic Pregnancy Foundation. Registered charity no 1122286. UK: Esher.

8. De Ott D. A case of unilateral tubal twin gestation. Ann Gynecol Obstet. 1891;36:304.

9. Eze JN, Obuna JA, Ejikeme BN. Bilateral tubal ectopic pregnancies: a report of two cases. Ann Afr Med. 2012;11(2):112-115.

10. Revicky V, Krishna A, Al-Taher H. Spontaneous monochorionic monoamniotic twin tubal ectopic pregnancy. J Obstet Gynaecol. 2009;29(5):447-448.

11. Brazao K, Frietas C, Farinha L. Spontaneous unilateral dichorionic twin tubal pregnancy. Acta Obstet Ginecol Port. 2011;5:193-197.

12. Karadeniz RS, Dilbaz S, Ozkan SD. Unilateral twin tubal pregnancy successfully treated with methotrexate. Int $J$ Gynaecol Obstet. 2008;102(2):171-178. 